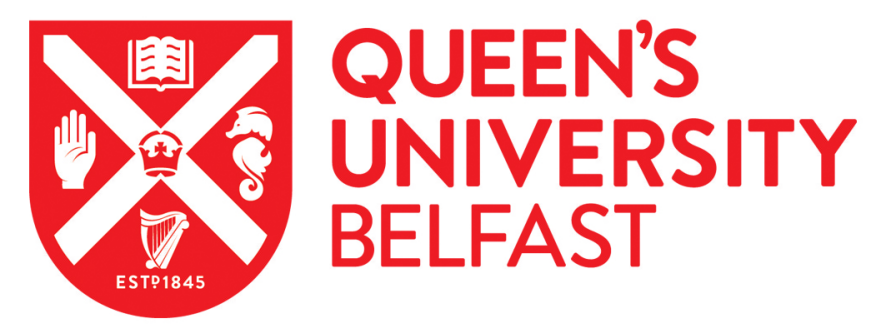

\title{
Chord length distribution to particle size distribution
}

Pandit, A. V., \& Ranade, V. V. (2016). Chord length distribution to particle size distribution. AIChE Journal. https://doi.org/10.1002/aic.15338

\section{Published in: \\ AIChE Journal}

\section{Document Version:}

Peer reviewed version

Queen's University Belfast - Research Portal:

Link to publication record in Queen's University Belfast Research Portal

\section{Publisher rights}

(c) 2016 American Institute of Chemical Engineers.

This is the peer reviewed version of this article, which has been published in final form at DOI:10.1002/aic.15338. This article may be used for non-commercial purposes in accordance with Wiley Terms and Conditions for Self-Archiving.

\section{General rights}

Copyright for the publications made accessible via the Queen's University Belfast Research Portal is retained by the author(s) and / or other copyright owners and it is a condition of accessing these publications that users recognise and abide by the legal requirements associated with these rights.

Take down policy

The Research Portal is Queen's institutional repository that provides access to Queen's research output. Every effort has been made to ensure that content in the Research Portal does not infringe any person's rights, or applicable UK laws. If you discover content in the Research Portal that you believe breaches copyright or violates any law, please contact openaccess@qub.ac.uk. 


\title{
Chord Length Distribution to Particle Size Distribution
}

\author{
Ajinkya V. Pandit \& Vivek V. Ranade* \\ Idustrial Flow Modeling Group (IFMG), \\ Chemical Engineering \& Process Development, \\ National Chemical Laboratory, Pune, India. \\ *vv.ranade@ncl.res.in
}

\begin{abstract}
A simple model is presented to extract the Particle Size Distribution (PSD) from the Chord Length Distribution (CLD) measured using a Focused Beam Reflectance Measurement (FBRM) probe. The model can be implemented using simple spread sheeting tools and does not require the description of additional parameters as opposed to previous models. The model was validated for two systems consisting of spherical ceramic beads by comparing model predicted PSD against the PSD obtained through image analysis (IA). Then, the model was evaluated by considering various systems consisting of irregularly shaped particles (sand/zinc dust/plasma alumina). Model predictions accurately predicted the mean but over-predicted the variance of the PSD in comparison with the PSD obtained from IA. However, overall, a reasonable agreement was observed. Finally, the model was shown to be accurate in predicting PSD in comparison with the measured PSD for systems of practical relevance such as for paracetamol and p-aminophenol crystals.
\end{abstract}

\section{Keywords}

FBRM, PSD, CSD, CLD, Crystallization, Paracetamol 


\section{Highlights}

- Developed a model to derive PSD from an FBRM measured CLD

- Validated the model for two systems of regularly shaped particles consisting of spherical ceramic beads

- The model was tested for various particle systems consisting of irregularly shaped particles: (a) sand (b) plasma alumina (c) zinc dust and was shown to be useful

- The model was then applied successfully to two particle systems of practical relevance: (a) paracetamol crystals (b) p-aminophenol crystals

- Model predicts PSD accurately using the FBRM CLD information requiring no additional parameters and using simple spread sheeting tools 


\section{Introduction}

Measurement of the Particle Size Distribution (PSD) of a given sample of precipitated solute in solvent is an important problem. The PSD of the sample is an important parameter in the crystallization industry to assess the quality of a sample. Measurement of the PSD also becomes important for modelling the kinetics of the nucleation and crystal growth processes. There are several techniques available to measure the PSD. The most popular ones are the Laser Diffraction and the Microscopy methods. These enable a complete characterization of the PSD curve however, are offline methods. Hence it is difficult to obtain PSD results using these methods for transient processes such as crystallization as we are limited to only investigating the 'equilibrated' samples. A nephelometer (or turbidity probe) is often used to study crystallization systems. However, even though it allows for the in-situ and online measurements, the results provided by this method do not give any information regarding the PSD.

The Mettler Toledo Particle Vision and Measurement (PVM) is another popular measurement device which extends the microscopy technique and enables the measurement of PSD in-situ and online.2 dimensional images of the particles as viewed from the probe window are recorded which are later processed to obtain the PSD. The Mettler Toledo Focused Beam Reflectance Measurement (FBRM) probe is also widely used to study crystallization. The FBRM probe works on the principle of laser back-scattering. A laser is emitted from a laser source inside the probe. The source is rotated around the probe axis such that the laser revolves remaining parallel to the probe axis. The laser traces a cylindrical surface around the probe axis inside the probe. A very high speed of rotation is used (in the present study the tangential speed of the source was set to $2 \mathrm{~m} / \mathrm{s}$ ). At the probe tip is a sapphire window which is in contact with the solution wherein the particle measurements are to be made. Whenever the revolving laser encounters a particle along its circular path it gets reflected back into the probe. Each instance of this reflection is treated as one count of particle detection and the duration of time for which the beam is reflected multiplied by the tangential speed of the laser becomes the length of the chord which the laser 'traces' on the particle surface. Thus, in course of time and in dense solutions, multiple particles are recorded by the FBRM probe and hence we obtain the number of counts and the particle chord length distribution data (CLD). 
The CLD measured by the FBRM probe, although related to the PSD, bears no obvious resemblance to the PSD. The CLD data needs to be interpreted in some way to obtain information regarding the PSD. Much work has been done recently to address this problem. Li et. al. (2013) ${ }^{1}$ developed an empirical method for the determination of the PSD from the CLD. Multiple fingerprint CLD's for known particle sizes were first meticulously determined over the size range of interest. The actual PSD was estimated as the one which yielded the same CLD as the measured CLD by a linear combination of the fingerprint CLD's. However, this method requires a lot of standardized data (fingerprint CLD's) in order to calibrate the model for a specific system before it starts to make predictions. Gathering this standardized data is not always possible and is definitely time consuming and cumbersome. Besides, the aforementioned publication, other efforts directed towards solving the CLD to PSD problem all follow a definite methodology. First the CLD of a single isolated particle (or equivalently a monodisperse system) is derived theoretically. Then, the CLD is derived theoretically for a system with a known PSD. Finally, the inverse problem of determining the PSD for a known CLD is addressed. Having established the first part, the second part is relatively straightforward. Several efforts have been directed to specifically address the first part of the problem. Li et. al. (2005) $)^{2,3}$ presented a model to determine the CLD for a spherical particle. The model was extended to allow for various particle shapes using a 2D super ellipsoidal function. However, the model only considered a fixed 2D particle projection to determine the CLD and did not account for the different particle orientations and the subsequent effect on the $2 \mathrm{D}$ projections. In the model presented by Ruf et. al. $(2000)^{4}$ all possible 2D projections of the 3D shape (with different orientations) were considered for determining the CLD. The approach to calculate the CLD from the 2D particle projection is fundamentally similar to the 2D model proposed previously.

The inverse problem of determining the PSD from a known CLD is more troublesome. Typically, the PSD is estimated iteratively by starting from a guess PSD and comparing the predicted CLD and the measured CLD to evaluate the direction of the iteration. However, due to the non-linear nature of the CLD expressions obtained from the single particle models, the inverse problem becomes an ill posed mathematical problem. The most popular methods include the Least Squares (LS) and the Constrained 
Least Squares (CLS) algorithms (Li et. al., 2005) ${ }^{2,3}$. However, when CLD measurements are noisy, as are typically observed for the case of crystallization systems, oscillations or negative values are obtained for the PSD’s using these methods. To overcome this pitfall, Li et. al. (2005) proposed an iterative NonNegative Least Squares algorithm for the inversion problem ${ }^{2,3}$. Worlitschek et. al. (2003) ${ }^{5}$ extended the model proposed by Ruf et. al. (2000) ${ }^{4}$ by considering the method of projection onto convex sets (POCS) to solve the inversion problem. Agimelen et. al. (2015) ${ }^{6}$ reported an algorithm to estimate the PSD from CLD measurements for needle shaped crystals. Appropriate single particle CLD models reported in literature were used and the algorithm required no additional information such as approximate particle size range or particle aspect ratio to constrain the iterative procedure as for previous efforts.

The methodology used in the present study also follows the definite approach used by previous authors wherein, first a single particle CLD model is proposed and later the inversion problem (deriving PSD from CLD) is addressed. However, it differs from previous approaches on two important fronts. First, the model for the single particle CLD is fundamentally different from the models reported in literature. This leads to a mathematically very simple expression for the CLD of a single particle. Second, instead of solving for all the number of particles in each bin of the discretized PSD, the PSD is assumed to be modelled by a suitable distribution function (Normal, Lognormal). This reduces the number of parameters to be iterated to only two: the mean and the variance of the distribution. As a result of both of these distinctions, the inversion problem becomes straightforward and can be solved using simple spread sheeting tools. The model was then validated for two systems consisting of spherically shaped ceramic beads. The model was then evaluated for three systems of irregularly shaped particle systems consisting of: (a) sand particles; (b) plasma alumina particles and (c) zinc dust particles. By irregularly shaped, it is implied that particle shape within the system is not uniform. The model was then successfully applied to two particle systems of practical relevance: (a) paracetamol crystals and (b) paminophenol crystals. The performance of the model was compared with the popular models reported by Li et. al. (2005) $)^{2,3}$ and Worlitschek et. al. $(2003)^{5}$. The model coupled with the FBRM measured CLD is a powerful tool to monitor the PSD even in transient processes such as crystallization.

\section{Mathematical Model}




\section{Single Particle Model}

As mentioned previously, the first problem that needs to be addressed is:

For a single particle of diamater $D$, what is the probability that a 'randomly cut' chord measures as having its length between $X_{1}$ and $X_{2}\left(\right.$ where $X_{1}$ and $\left.X_{2}<D\right)$ ?

This problem statement is similar to the one proposed by Bertrand, in the Bertrand's Paradox (a classic paradox in probability theory). It is important to note that the important variable in the problem is the length of the randomly cut chord rather than identifying a specific random chord itself. The key issue in this problem is the interpretation of the 'randomly cut' chord. The length of a random chord may be determined in three ways (as stated in the paradox):

(a) Method 1: One point may be arbitrarily fixed on the circle and the other may be chosen at random along the circumference of the circle. The chord joining the two points is then the random chord and the length depends upon the position of the second point in relation to the first point.

(b) Method 2: The distance of the chord from the centre of the circle is enough to specify its length. This distance may then vary from 0 to the (known) radius of the circle. The length may then be calculated once a random value is assigned for the distance of the chord from the center of the circle.

(c) Method 3: For every point within the circle, there is a chord such that the considered point is the midpoint of the chord. So, the problem of randomly chosing a chord may then be equated to randomly chosing a point within the circle. The length may then be determined once the poistion of the point is known.

In the paradox, it is demonstrated that the different interpretations of the randomness of the chord lead to different results. The classical solution of the paradox is that there is no 'right' solution. An appropriate definition of randomness must be chosen depending upon the physcial scenario considered to obtain an appropriate answer. More information regarding the Bertrand Paradox is provided in the Supplementary Information. The FBRM views a 2D projection of the particle. Methods 1 and 2 provide 
a 1D solution (random variable along the radius or the circumference of the circle) to the problem and it may be inferred that information is lost regarding the orientation of the chord with respect to a fixed frame of reference (for instance the FBRM probe). Method 3 is the only method that addresses the 2D nature (area of the circle) inherent in the physcial system considered. Previous researchers have used a methodology parallel to Method 2. However, in the present text, it is argued that Method 3 is a more accurate interpretation to determine the length of a randomly cut chord for the case of a FBRM than Methods 1 or 2.

The laser views a 2-D projection of the particle. Considering the case of a spherical particle (of diameter ' $D$ '), the 2D projection is a circle with the same diameter $D$. Now, it should be noted that for every point inside the circle, there is associated with it, a unique chord for which the considered point is the midpoint of the chord.Thus, chosing a random chord of a circle is equivalent to choosing a random point inside the circle.The locus of the midpoints of the chord of the same length is a circle with dimensions as shown in Figure 1(a). Any point on the circle (dotted line) corresponds to a chord with length $x$ and every chord with length $x$ will have its midpoint on the circle (dotted line). For a chord whose length is between $x$ and $y$, it is necessary that its midpoint should lie in the shaded area given in Figure 1(b).

The probability of measuring a chord with size between $x$ and $y(x<y)$, or $P(x, y, D)$ becomes the ratio of the area of the shaded region to the total area of the circle:

$P(x, y, D)=\frac{y^{2}-x^{2}}{D^{2}}$

Since the probability, that a measured chord has a size less than or equal to the particle size, is unity and also it is clear from direct substitution into Equation (1) that:

$P(0, D, D)=1$

It is worth noting that for the probability function defined here, the following property holds $(y<z)$ :

$P(x, y, D)+P(y, z, D)=P(x, z, D)$ 
Also, since no chord length greater than the diameter can be measured, the following definition must be made for all $z>D$ :

$P(D, z, D)=0$

\section{Particle Size Distribution to Chord Length Distribution}

Particle size distributions and chord length distributions are typically represented in the form of histograms. The entire relevant span of the particle size co-ordinate is divided into discrete 'bins'. The bin sizes, typically, are either uniform or increase logarithmically starting from the first bin, depending upon how the measurements were made or what convention was adopted. The histogram plots the frequency, or number of particles, as is applicable in the present study, that are recorded in each of these bins.

\section{CLD for a monodisperse collection of particles}

The histogram for the PSD of a collection of monodisperse particles is represented by a single peak. The location of the peak corresponds to the bin representing the size of the monodisperse particles. In a normalized form, the value of the peak will be unity. A normalized PSD for a collection of monodisperse particles of size $95 \mu \mathrm{m}$ is shown in Figure 2. The bins are considered to be uniformly sized having width equal to $10 \mu m$ and the domain considered extending from 0 to $150 \mu m$. It is useful to define a representative size, wherein, each particle contained in the bin can be assumed to be of the corresponding representative size. The histogram shown in Figure 2 is plotted versus the representative bin size.

In the previous section the problem of determining the probability of measuring a chord within a particular size range, say for instance between $x$ and $y$, for a single particle was addressed. Now, provided that the number of particles is large enough, the aforementioned probability can be equated to 
the fraction of the total number of particles (for a monodisperse system) which would be measured as having chords between sizes $x$ and $y$. This important assumption allows the determination of the CLD from a single particle model and has been reported by other researchers aswell ${ }^{2,4}$. The assumption is a statistical assumption in the sense that it gains validity as the sample size of the number of particles recorded increases and can be assumed to be true when the number of particles is sufficiently high. It is expected that the number of particles recorded by the FBRM are sufficiently high enough that the aforementioned assumption is valid.

Suppose the particle size co-ordinate is distributed into $k$ bins. Let $L_{i-1}$ and $L_{i}$ denote the lower and upper boundaries of the $i^{\text {th }}$ bin. $L_{0}$ will typically be set to zero. Let the number of particles in the $i^{\text {th }}$ bin be denoted as $N_{i}$ and the representative size of the $i^{\text {th }}$ bin (defined as the midpoint of the boundary points of the $i^{\text {th }}$ bin), by $X_{i}$. For the present case, let us assume that all the monodisperse particles are contained in the $m^{\text {th }}$ bin. Let the total number of particles in the system be denoted by $N_{T}$. Let the number of particles measured in the $i^{\text {th }}$ bin for a general PSD be $N_{i}$ and for a CLD be $M_{i}$. Let the fraction of the total particles (contained in the $m^{\text {th }}$ bin) measured as having chords in the $i^{\text {th }}$ bin, be denoted by $A_{i, m}$. As defined in the previous section, the probability of measuring a chord in the $i^{\text {th }}$ bin for a particle belonging to the $m^{\text {th }}$ bin can be written as $\mathrm{P}\left(L_{i-1}, L_{i}, L_{m}\right)$. It should be noted, that the particle size here was taken as the upper boundary $\left(L_{m}\right)$ rather than the representative bin boundary $\left(X_{m}\right)$. This is done so as to make the calculations more lucid and as will be demonstrated later by extensive validation, does not affect the model applicability.

From the assumption that the fraction of total particles (contained in the $m^{\text {th }}$ bin) measured as having chords in the $i^{\text {th }}$ bin is equal to the probability of measuring the chord in the $i^{\text {th }}$ bin for a single particle belonging to the $m^{\text {th }}$ bin, we may write the following equality:

$A_{i, m}=\frac{M_{i}}{N_{m}}=P\left(L_{i-1}, L_{i}, L_{m}\right)$

Here, $i \leq m$. As discussed in the previous section, if $i>m$, then $A_{i, m}$ is equal to zero. It should be noted that for a monodisperse system of particles contained in the $m^{\text {th }}$ bin, the the total number of particles is the number of particles contained in the $m^{\text {th }}$ bin and hence: 
$N_{m}=N_{T}$

From Equation (5), the number of particles in the $i^{\text {th }}$ bin in the CLD may be written as:

$M_{i}=P\left(L_{i-1}, L_{i}, L_{m}\right) N_{m}$

The number of particles represented by $M_{i}$ is the required CLD. For a system of monodisperse particles (belonging to a single bin: $m^{\text {th }}$ bin), the normalized CLD can be obtained by dividing $M_{i}$ with the total number of particles. For a sample case of a collection of particles contained between the 90 to $100 \mu \mathrm{m}$ size range, the normalized chord length distribution obtained using the above described methodology is shown in Figure 2. A uniform bin size of $10 \mu \mathrm{m}$ and a domain ranging from 0 to $150 \mu \mathrm{m}$ are considered. The total number of particles may also be obtained by summing the number of particles in each bin in the CLD. Thus we have:

$\sum_{i=1 \text { to } m} A_{i, m}=1$

Hence, all the particles considered are accounted for. In the next section, a procedure to obtain the CLD from a given PSD is discussed.

\section{CLD for a known PSD}

A PSD is a histogram which plots the number of particles measured versus the size ranges or the bins in which they are measured in. It is similar to the CLD with the difference that the size ranges correspond to the actual sizes (as defined appropriate) of the particles rather than chord sizes. The monodisperse system discussed in the previous section is a special case where the sizes of the particles are limited to just one bin. A more general PSD will contain particles in many of the bins considered in the domain similar to the CLD obtained in the previous section. Now, the only difference between the monodisperse case and the present case is that instead of the CLD being computed only from one bin (like the $m^{\text {th }}$ bin considered in the previous study), all the bins considered in the general PSD will contribute to the bins of the CLD. So, the problem of converting a PSD into a CLD is reduced to counting the contributions of each of the bin of the PSD towards each of the bin of the CLD and adding them up. With this in mind, we define a new variable $M_{i, j}$ to be the number of particles whose actual size corresponds to the 
$j^{\text {th }}$ bin, being counted as having a chord size which corresponds to the $i^{\text {th }}$ bin. With this definition, in the previous section the value of $M_{i}$ calculated actually corresponds to $M_{i, m}$ in the new notation. The notation $M_{i}$ retains its definition, of being the total number of particles in the $i^{t h}$ bin in the CLD, although now it has a deeper meaning as being the sum of the contributions from all the different bins, rather than just from an arbitrary $m^{\text {th }}$ bin.

Now, the contribution of, say the $j^{\text {th }}$ bin of the PSD, towards the $i^{\text {th }}$ bin of the CLD, in terms of the number of particles, may be calculated using the analysis presented in the previous section. Thus, keeping in mind the new variable definitions, we may write the following equality:

$M_{i, j}=P\left(L_{i-1}, L_{i}, L_{j}\right) N_{j}$

The total number of particles in each bin can be calculated by simply summing all the contributions of the different bins.

$M_{i}=\sum_{j=i \text { to } k} M_{i, j}$

Where, $\mathrm{k}$ is the total number of bins. The bins are summed starting from the present bin index. This is because the contribution of the bins from the PSD with a size smaller than the present bin towards chords being measured in the present bin will be zero. The values $M_{i}$ are the required CLD.

Also, since we are counting all of the particles, it is interesting to note and it may also be rigorously derived that the total number of particles is in fact conserved. Hence,

$N_{T}=\sum_{i=1 \text { to } k} M_{i}$

The normalized CLD may be obtained by dividing the CLD with the total number of particles. Let $A_{i}$ denote the fraction of the total particles being measured as a chord in the $i^{\text {th }}$ bin. Then we may write the following relation:

$A_{i}=M_{i} / N_{T}$

The Inverse Problem 
Several methods are proposed to solve the inverse problem of calculating the PSD from the CLD ${ }^{2,5,6}$. In the present study, a simple iterative method starting from a guess PSD is used. A guess PSD represented in the form of a 2 parameter normal or lognormal distribution function is considered and the corresponding CLD is determined using the PSD to CLD model described in the previous section.

A two parameter normal distribution function is represented by the following function:

$f(x)=\frac{1}{\sigma \sqrt{2 \pi}} e^{\frac{-(x-\mu)^{2}}{\sigma^{2}}}$

The two parameter lognormal distribution is represented by the following expression:

$f(x ; \mu, \sigma)=\frac{1}{2 x \sigma \sqrt{2 \pi}} e^{-\frac{(\ln x-\mu)^{2}}{\sigma^{2}}}$

In the above equation, the two parameters $\mu$ and $\sigma^{2}$ stand for the mean and the variance of the distribution functions. It is known that this function arises in various types of naturally occurring distributions and hence, it should also be sufficient to describe the distribution considered in the present study. To obtain the corresponding PSD, first, the bin boundaries and the representative sizes of each bin need to be finalized. The number of particles in each bin $N_{i}$ is then the value of the respective distribution function evaluated at $X_{i}$. For any sort of distribution function to be valid the following equality must hold:

$\int_{0}^{\infty} f(x) d x=1$

However, because of discretizing the domain, the area of the discrete PSD will not be unity. Hence, we need to correct for this to avoid any errors. This is done by reassigning the number in each bin as follows:

$N_{i, \text { corrected }}=\frac{N_{i}}{\sum_{i} N_{i}\left(L_{i}-L_{i-1}\right)}$

These new corrected values were now referred to as $N_{i}$.

The values for the 2 parameters (mean and variance) required for the full specification of either the normal or the lognormal distribution can be determined by optimizing the $R^{2}$ error ( $R^{2}$ should be closest 
to 1) between the measured and the model predicted CLD. A simple multi parameter optimization routine available with most spread sheeting tools is sufficient for these purposes. The algorithm for this process is given in Figure 3.

To quantify the error between the derived CLD and the measured CLD, the coefficient of determination or $R^{2}$ was used. The $R^{2}$ value can be calculated as follows:

$$
\begin{aligned}
& \bar{M}=\frac{1}{k} \sum_{i=1 \text { to } k}\left(M_{i, \text { experiment }}-M_{i, \text { model }}\right) \\
& S S_{\text {tot }}=\sum_{i=1 \text { to } k}\left(M_{i, \text { exp }}-\bar{M}\right)^{2} \\
& S S_{\text {res }}=\sum_{i=1 \text { to } k}\left(M_{i, \text { model }}-M_{i, \text { exp }}\right)^{2} \\
& R^{2}=1-\frac{S S_{\text {res }}}{S S_{\text {tot }}}
\end{aligned}
$$

\section{PSD measurement through Image Analysis}

Before presenting model results, it is worthwhile to spend some time on how the PSD may be obtained through image analysis. The image analysis procedure for determining the particle sizes is outlined in Figure 4. A series of 2D images were taken for the given system of particles under a microscope. The images were then converted into binary images (having just 2 colours: black and white). The binary images further treated to fill the holes inside the particles, remove any overlapping particles, separate touching particles, remove particles on the image boundary which were incomplete and remove the background noise to obtain reliable data. These binary images were then used to identify particles. The image pixel size in terms of pixel/ $\mu m$ is known from image calibration. From the last stage of image analysis, and the known pixel/ $\mu m$ ratio from calibration, the visible area of the particles within one image can be calculated in $\mu m^{2}$. In the present study, after this area is obtained, the spherically equivalent diameter was obtained for each particle which is defined as the diameter of a sphere having the same visible cross sectional area as the visible area obtained for a particle using image analysis. This spherically equivalent diameter was obtained for a series of particles. The PSD may be obtained by 
specifying the bin spacing to be used and counting the number of particles that fall within each size range from the data obtained in the previous step.

The processing of images was done using the ImageJ software.

\section{Results \& Discussions}

\section{Model Validation for Regularly shaped particles}

The above presented model was first validated for a system of regularly shaped particles. By regularly shaped, it is implied that the particles considered in the present section were nearly spherical. The PSD was available for a calibration sample used for the FBRM probe. The FBRM probe calibration sample consisted of nearly perfect spherical ceramic particles. Shown in Figure 5 (a), is an image of the particles taken using a PVM. It can be assumed that the particles are spherical in shape. Based on the images captured by the PVM, a PSD was prepared for the lengths and widths measured for different particles obtained from image analysis. The PSD is shown in the Figure 5 (b).

A cumulative PSD, obtained by summing the individual length and width distributions, was considered as the PSD of the sample. The cumulative PSD was then normalized by division with the total number of particles to enable comparison with the calculated PSD. The normalized PSD obtained is shown in Figure 6 (b). The PSD was obtained through the image analysis done on a random sample size of 600 particles. The corresponding CLD of the calibration sample was also known. The CLD was also normalized by dividing the CLD with the total number of chords measured. The presented model was applied to extract the PSD from the measured CLD.A two parameter normal distribution function was used to represent the PSD. A uniform bin size of $10 \mu \mathrm{m}$ with a domain spanning a range of 0 to $300 \mu \mathrm{m}$ was used corresponding to the type of data available from PSD/CLDmeasurements. All the distributions considered were normalized by dividing with the respective total number of particles/chords, as was relevant, to enable comparison. The 2 parameter values (mean and variance) for representing the PSD were obtained by fitting the normalized CLD obtained from the present model with the normalized CLD measured using FBRM. A comparative plot between the two is shown in Figure 6 (a). The $R^{2}$ value of 
for the fit was 0.92 . The $R^{2}$ value obtained is quite low. As can be seen from the Figure, there is a significant mismatch between the model-predicted CLD and the experimental CLD especially in a size range less than $10 \mu m$.The comparison between the normalized PSD measured experimentally and the normalized PSD predicted using the model is shown in Figure 6 (b).

As can be seen in figure, the model accurately predicts the PSD using just the CLD information.

The model was then validated against another data set for regularly shaped particles. The data reported by Li et. al. (2005) $)^{3}$ was used for the model validation. An image of the ceramic bead particles used by Li et. al. (2005) $)^{3}$ is provided in the cited work. It was seen from the image, that the particles were nearly spherical and could be classified as regularly shaped particles. A two parameter normal distribution function was used to model the PSD. The PSD for the sample of ceramic particles was extracted from the CLD measured using the FBRM probe (reported by Li et. al. $(2005)^{3}$ ). The 2 parameter values for representing the PSD were determined by fitting the normalized model CLD to the normalized experimentally measured CLD using an optimization routine to tweak the mean and the variance parameters such that the $R^{2}$ value is closest to unity: a condition for complete match. For the present data set, an optimized $R^{2}$ value of 0.9794 was obtained corresponding to the fit between the normalized experimental and normalized model CLD's shown in Figure 7(a). A comparison between the normalized PSD measured using Image Analysis (reported by Li et. al. $(2005)^{3}$ ), the normalized PSD obtained using the model presented by Li et. al. (2005) ${ }^{3}$ and the normalized PSD obtained using the present model is shown in Figure 7(b). As can be seen from Figure 7(b), the normalized PSD's obtained using the present model is in excellent agreement with the PSD obtained through image analysis. The PSD obtained using the Li et. al. (2005) ${ }^{3}$ model also predicts the PSD accurately.

From these two cases, we may conclude that the model gives accurate PSD information for regularly (spherical) shaped particles. In the next section we discuss the model validity for the case of irregularly shaped particles.

Evaluation of Model for Irregularly Shaped Particles 
In this section, the model validity for different systems of irregularly shaped particles is considered. By irregularly shaped, it is implied that no specific shape or similarity of shape can be established within the system of particles considered.

\section{Sand Particles}

Normal sand procured at a local dealer was first passed through a sieving tower. The sand trapped within the sieve ranges of $50 \mu \mathrm{m}$ to $103 \mu \mathrm{m}$ was considered for the experiment. Around $8 \mathrm{~g}$ of sand was taken and it was washed with sulphuric acid to remove any organic impurities. After this, it was repeatedly washed using distilled water over a 50 $\mu m$ sieve to remove any particles or impurities smaller than $50 \mu m$. The sand was charged into a $250 \mathrm{ml}$ reactor along with $200 \mathrm{ml}$ of distilled water. The temperature of the mixture was maintained at $25^{\circ} \mathrm{C}$. The FBRM probe was inserted into the reactor to record the chord length distribution. The solution was stirred using a pitched blade down flow impeller with a stirring speed (800 RPM) which was sufficiently high enough to ensure that the mixture was well-mixed and well suspended. The sufficiency of the stirring speed to ensure well-mixed conditions was verified by increasing the stirrer speed gradually until a point where the number of counts recorded by the FBRM probe did not increase with increasing impeller speed. The CLD of the sample was recorded for a period of 10min using the FBRM probe under these conditions.

The sand particles were then separated from the solution and the PSD was determined by observing the particles under a microscope (Image Analysis). A sample image taken under a microscope for the present case is shown in Figure 8 (a). A binary image was obtained through image processing of the sample image as shown in Figure 8 (b). The PSD was determined by processing the sizes of 380 particles. The normalized PSD obtained from Image Analysis is shown in Figure 9 (b).

From image analysis, it was observed, that although the particles were separated using a sieving tower, there were debris particles which had sizes less than $10 \mu \mathrm{m}$. Now, even though they were visible under a microscope, characterizing their size was not possible. The minimum particle size from the measured sample recorded through image analysis was $16 \mu \mathrm{m}$. The presence of debris particles is also indicated by the CLD measured by the FBRM as shown in Figure 9 (a). In the normalized CLD shown in Figure 
9 (a), two peaks can be seen, one corresponding to a size less than $10 \mu \mathrm{m}$ and another around the 60 to $70 \mu m$ mark. This suggests that the PSD of the present sample is actually a bimodal distribution. Now, in the present study, the PSD is modelled as a unimodal distribution. Hence, the resulting CLD is also unimodal. The approach may be extended easily for the case of bimodal distributions. For a bimodal distribution, the function would be modelled as a sum of two independent lognormal or gaussian distribution functions. The bimodal distribution will then be characterized using 4 parameters: the mean and the variance parameters of both distributions. The CLD may be obtained from the PSD by the procedure discussed in the manuscript. For the inverse problem, starting from a guess PSD, the 'true' PSD may be obtained by an iterative procedure similar to the case of the inverse problem of a unimodal distribution. However, in case of two parameters (as in the unimodal case), there will be 4 parameters which need to be iterated.

In the present case, the PSD for particles lower than $10 \mu \mathrm{m}$ could not be obtained reliably through image analysis. Hence, instead of considering a bimodal distribution, the model was selectively evaluated for bigger sized particles (more than 10 $\mathrm{mm}$ ) using a two parameter (mean and variance) unimodal log normal distribution. To do this, the square weighted CLD is considered instead of the normal CLD. The square weighted CLD is used to specifically analyze the distribution of bigger sized particles in collection particles which span different size ranges. The square weighted CLD may be obtained by multiplying the number of particles in each bin by the square of the respective representative bin size. The square weighted CLD can be normalized by dividing each of the resulting terms in the bins by the sum of resulting terms in each bin spanning the entire domain. A comparison between the normalized square weighted CLD and the normalized CLD for the system of sand particles considered is shown in Figure 9 (a). As can be seen, the normalized square weighted CLD tracks the distribution among the bigger sized particles and the lower sized particles are essentially ignored.

To determine the PSD, the $R^{2}$ value for the fit between the normalized square weighted CLD's obtained using the FBRM probe and through model was optimized to be close to unity. For the present case, an $R^{2}$ value of 0.998 was obtained. The respective comparison between the normalized square weighted CLD's obtained experimentally and the one used in the model is shown in Figure 9 (a). The comparison 
between the normalized PSD obtained through image analysis and from the model is shown in Figure 9 (b). Logarithmic bin spacing was used to calculate the PSD such that there are 100 bins which span a domain starting from 1 to $1000 \mu m$ and a bin size of 0.0715 for the first bin. As can be seen from Figure 9 (b), the model is able to predict the mean of the PSD very well; however, the variance or the spread parameter of the PSD was not predicted well. The actual distribution is seen to be narrower than the one obtained using the model. However, we may conclude that the agreement is reasonable. A discussion on the possible reasons for the discrepancy is presented later is this section after considering other similar cases for systems of irregularly shaped particles.

\section{Plasma Alumina}

The validity of the presented model was tested for a system of plasma alumina particles. An image of the plasma alumina particles used by Li et. al. $(2005)^{3}$ is provided in the cited work. As was seen from the image, similar to the case of sand particles, there was no similarity in shape that can be inferred. A two parameter (mean and variance) lognormal distribution was considered to model the PSD. The PSD for the present case was derived by fitting the normalized model CLD with the normalized CLD measured using the FBRM probe (reported by Li et. al. $(2005)^{3}$ ) such that the $R^{2}$ value of the deviation is closest to unity. The $R^{2}$ value for the present case was found to be 0.9947 and the corresponding comparison between the normalized model CLD and the normalized FBRM measured CLD (reported by Li et. al. $(2005)^{3}$ ) is shown in Figure 10 (a). A comparison between the normalized PSD obtained from Image Analysis (reported by Li et. al.), and the normalized PSD’s obtained using the present model and the best prediction using the model presented by Li et. al. (2005) ${ }^{3}$ is shown in Figure 10 (b). As can be seen from Figure 10 (b), similar to the case of sand particles, the present model accurately predicted the mean of the PSD but could not capture the spread of the PSD. The PSD predicted using the model presented by Li et. al. (2005) ${ }^{3}$ shows a good agreement for larger size particles apart from reasonably predicting the mean. This is because the model presented by Li et. al. $(2005)^{3}$ allows for the description of an additional 'particle shape factor'. The value of the 'shape factor' can be determined by either fitting model predictions to both the CLD and the PSD or through information known before hand about the particular system. 


\section{Zinc Dust}

The validity of the present model is tested against another system of irregularly shaped particles: zinc particles. An image of the zinc dust particles used by Li et. al. $(2005)^{3}$ is provided in the cited work. As was seen in the image, no pattern could be inferred regarding the shape of the particles. A two parameter (mean and variance) lognormal distribution was considered to model the PSD. The parameter values for mean and variance were obtained by fitting the normalized model derived CLD with the normalized CLD measured using the FBRM (reported by Li et. al. $(2005)^{3}$ ). A $R^{2}$ value of 0.976 was obtained for the fit. A comparison between the measured normalized CLD's obtained by, fitting and measurement using the FBRM, is shown in Figure 11(a). The PSD for the sample was reported by Li. et. al (2005) which was measured using Image Analysis. A comparison between the normalized PSD reported by Li et. al. and the normalized PSD's obtained using the present model and the model presented by Li et. al. $(2005)^{3}$ is shown in Figure 11(b). As can be seen from Figure 11(b), the model predictions from the present analysis, predicts the mean of the PSD reasonably well, however, as in previous cases of irregularly shaped particles, the variance is not predicted accurately. The PSD predicted by the model presented by Li et. al. (2005) ${ }^{3}$ agrees well with the experimental data apart from the additional particles predicted in a size range below $50 \mu \mathrm{m}$. As for the previous case, the better agreement can be attributed to the use of an additional 'shape factor'. For irregularly shaped particle systems, it was consistently observed that the mean of the PSD predicted by the model was in reasonable agreement with the experimentally measured mean, while the variance was not. This mismatch was also observed for the model presented by Li et. al. (2005) ${ }^{3}$. For irregularly shaped particles, there arise 2 ambiguities. Firstly, how to define the particle size for an irregularly shaped particle and thereafter find the particle size distribution experimentally. Secondly, from the point of view of the model, the question of determining the CLD for an irregularly shaped particle system becomes important. The particle size in the present study was defined as the equivalent diameter of a circle which represents the same area as the projected area of the particle visible through the microscope. Due to such a definition, the orientation of the particle with regards to the microscope has an important role in determining particle size and the particle size distribution. Although how this impacts the particle size distribution and what is the range of 
uncertainty introduced by it is not entirely clear. However, from the findings reported in the manuscript, what we can say about such systems is that the mean can be accounted for accurately using the model.

\section{Application of Model for Real Systems}

In the previous sections, we evaluated the model validity for a system of particles which are regularly and irregularly shaped. It was concluded that the model could accurately predict the PSD for the case of regularly shaped particles and could predict the PSD for irregularly shaped particles reasonably well. In the present section, the focus is on applying the model for two sets of particles which are practically relevant.

\section{Paracetamol Crystals}

In the present section, the model was applied for a system of Paracetamol crystals. This system is a popular subject for crystallization studies besides its practical relevance. For the purposes of model validation, the previous work done by Worlitschek, Hocker and Mazotti (2005) ${ }^{5}$ was considered. In the work presented, the authors have used a method of Projection onto Convex Sets (POCS) for the problem of restoration of a PSD from a measured CLD. For the validation of the POCS method, they have considered a system of $1 \%$ vol. acetaminophen (Paracetamol) in a toluene solution. The CLD data was interpreted using the POCS method to obtain a restored PSD. The restored PSD was then compared with the other offline methods to obtain a sample PSD: laser diffraction and TSI Aerosizer. The method was seen to adequately predict the particle size distribution.

In the present study, the validity of the present model for a system of paracetamol crystals is evaluated by deriving the PSD from the experimental CLD data and comparing the model predicted PSD against experimental PSD measurements. The experimental measurements of the CLD and the PSD for this section, used in the present study, were previously reported by Worlitschek et. al. (2005) . $^{5}$

An image of the Paracetamol crystals used by Worlitschek, Hocker and Mazotti (2005) ${ }^{5}$ isgiven in the cited work. The CLD measurements were noisy. The CLD measurements are noisy when the sample being measured has a low particle concentration. For very high particle concentrations, the CLD 
measurements have negligible noise and do not need smoothening. Typically, the presented model works well for high particle concentrations as the model predicted CLD may be compared directly against the measured CLD. For low particle concentrations, the noisy CLD can be smoothened by averaging out the CLDs measured over a brief time interval. However, the CLD reported by Worlitschek et. at. (2005) $)^{5}$ was an instantaneous CLD. The noise in the CLD is representative of the finite sample measured by the FBRM. Smoothening in effect cancels out this dependency on 'local' samples and is representative of the entire particle system and does not affect the CLD to PSD conversion. In the present study, the restored CLD data provided by Worlitschek et. al. (2005) , which is essentially a smoothened representation of the raw CLD data was considered. A comparison between the raw CLD data and the restored CLD reported by Worlitschek et. al. (2005) ${ }^{5}$ is given in the cited work.

A two parameter lognormal distribution was considered to model the PSD of the present system. The parameter values for the PSD were obtained by fitting the CLD obtained using the present model to the CLD measurements reported by Worlitschek et. la. (2005) $)^{5}$ A comparison between the normalized CLD obtained using the present model and the measured CLD (restored) reported by Worlitschek et. al. (2005) $)^{5}$ is shown in the Figure 12 (a). The data was obtained in terms of 360 logarithmically spaced bins ranging from sizes of 1 to 1000 microns. The $\mathrm{R}^{2}$ value of the fit was 0.996 . The values for the mean and variance were obtained to be 3.65 and 0.65 respectively.

To enable comparison of the presented derived PSD data with the measured PSD data reported by Worlitschek et. al. (2005) ${ }^{5}$, two types of distributions derived from the PSD need to be defined. Firstly the discretized length weighted logarithmic distribution $n_{1, l n}$ and secondly the discretized volume weighted logarithmic distribution $n_{3, l n}$. For an arbitrary $j^{\text {th }}$ bin, the following can be shown to be true:

$$
\begin{aligned}
& n_{1, l n}\left(X_{j}\right)=\frac{X_{j} N_{j}}{\mathrm{~B}_{1} \mathrm{w}_{\mathrm{s}}} \\
& n_{3, \ln }\left(X_{j}\right)=\frac{X_{j}^{3} N_{j}}{\mathrm{~B}_{3} \mathrm{w}_{\mathrm{s}}}
\end{aligned}
$$


Where $w_{s}$ is the logarithm of the ratio of expansion of the bins and for the present case has the value 0.0192. The other symbols $B_{1}$ and $B_{3}$ stand for the first and the third moments of the discretized PSD. The general $i^{\text {th }}$ moment of a discretized PSD may be determined by using the following relation:

$B_{i}=\sum_{j} X_{j}^{i} N_{j}$

A comparison of the discretized length weighted logarithmic distribution between the PSD calculated from the present study, the data obtained using the POCS method and from the TSI Aerosizer is shown in Figure 12 (b).

A comparison of the discretized volume weighted logarithmic distribution between the PSD calculated from the present study, the data obtained using the POCS method and from the TSI Aerosizer is shown in Figure 12 (c). As can be seen from both the figures, the present model yields reasonable comparison with methods for offline determination of PSD. In the volume weighted distribution for the PSD, there is an abruptness at the end because no bin sizes larger than 150 micron were considered for calculation.

Thus, we can conclude that even with the assumption of spherically shaped particles, the present model is seen to predict useful results. The present model is thus valid for predicting the PSD from the FBRM measured CLD for a system of paracetamol crystals.

\section{Para-Aminophenol Crystals}

Para-Aminophenol is a commercially important chemical (used in the manufacturing of paracetamol) which is obtained through crystallization. In the present study, the validity of the present model is tested for a system of p-Aminophenol crystals in water. Water was chosen as a solvent as p-Aminophenol is sparingly soluble in water. An industrial sample of p-Aminophenol was considered for analysis. An image of the p-Aminophenol crystals in water is shown in Figure 13(a). As can be seen from the Figure, there is a lot of debris (smaller crystals) apart from the bigger crystals. This suggests that this considered sample PSD might actually be bimodal. This is confirmed by the CLD measurements shown in Figure 13(b). The average chord size in the lower size range of the bimodal distribution shown in Figure 13(b), appears to be below $10 \mu \mathrm{m}$. The PSD of the Para-Aminophenol crystals was determined in the present 
study through Image Analysis of images obtained using a microscope. The minimum size recorded using Image Analysis for the present system was $29 \mu \mathrm{m}$. Sizes below this size range could not be resolved using image analysis. This is similar to the problem of validation for sand particles covered in one of the previous sections. As done for the case of sand, since, out of the bimodal distribution, we have the PSD data only for the larger size class, the model validity was tested only within the larger size range.

A unimodal template PSD represented using a two parameter log normal distribution, as done in the previous sections, was considered. In the model discussed in the present work, the values for the two parameters (mean and variance) of the PSD are obtained by fitting the model predicted CLD to the experimental CLD. A square weighted CLD, which may be derived from the CLD by a procedure mentioned in the section dealing with sand particles, gives preference to larger size particles. The smaller size particles are apparently artificially filtered from the data. The values of the two parameters of the PSD were instead obtained by fitting the model predicted normalized squared CLD to the experimentally measured (using FBRM) normalized squared CLD. A comparison between the fitted model predicted normalized squared CLD and the experimentally measured normalized squared CLD is shown in Figure 13(b). The $R^{2}$ value for the fit was found to be 0.997 . The PSD was measured using the Image Analysis procedure discussed earlier. A sample of 380 particles was measured. A comparison between the model-predicted normalized PSD and the normalized PSD obtained through Image Analysis is shown in Figure 13(c). The model is seen to slightly under-predict the mean particle size however, accurately accounts for the variance. It may be concluded that the presented model accurately predicted the PSD for the case of Para-Aminophenol crystals.

\section{A Word on Model Applicability}

A comparison between the expected values of the diameter (mean diameter) and the standard deviations for each of the distributions reported in the present study for different systems is given in Table 1 . The model was seen to predict good results for the case of spherical or regularly shaped particles. This was encouraging but expected as the model was fundamentally based on the assumption that the particles 
are spherical in shape. The model, when applied to a system of irregularly shaped particles, was seen to predict the mean accurately but over predict the standard deviation. This observation was consistent across all the three systems considered in this category. The discrepancy in the predictions points to the fact that the information about the shape is important. However, the shape itself varies within such systems. So, any model, built using a uniform shape assumption will face such limitations while predicting the PSD for an irregularly shaped particle system. For instance, even the model presented by Li et. al. $(2005)^{2,3}$, which allows the particle shape to be ellipsoidal and provides an adjustable parameter to tune the particle shape specific to a system, failed to capture the standard deviation accurately for the system of plasma alumina particles. Then, two systems of particles which are the potential application areas for the presented model were considered. The present model was seen to reasonably predict the PSD for a system of paracetamol particles as also for the case of p-aminophenol particles. Thus, even though, the model assumes a spherical particle shape, it still accurately predicts the PSD. The reason for this could be that the particles within a system of crystals are uniformly shaped as opposed to the shape variation which is typically seen in irregularly shaped systems.

A word of caution is necessary while using the present model. This does not have to do with the model itself but more with regards to the limitations in determining the CLD for particular systems using the FBRM. Sometimes, like in the case of glass particles, air bubbles, liquid-liquid emulsions and such, the optical properties of the particle/bubble/drop become important. The CLD then depends upon, whether and to what extent, the laser gets reflected back from the particle surface. The present model does not account for the optical properties of the particles and is not fit for use for systems with transparent particles/bubbles/drops. For applications related to such systems kindly refer to Kail et. al. (2009) ${ }^{7}$. Another difficulty arises where in due to inadequate suspension, larger particles tend to stay at the bottom of the vessel. In such cases, the CLD is measured for only the suspended, smaller sized particles. This results in an under prediction of the PSD. Adequate care should be taken that the particles are always uniformly suspended. In any case, it is good practise to validate the model once, against results from image analysis or other offline sizing techniques, while considering a particle system other than the one reported in the present study. 


\section{Conclusion}

In the present study a simple model was presented to derive the Particle Size Distribution (PSD) from a Chord Length Distribution (CLD) measured by the FBRM probe. The model does not require the specification of any parameter and enables the direct calculation of the PSD from known CLD measurements using simple spread sheeting tools. The model was first validated for two systems of regularly shaped (spherical) particles both consisting of ceramic beads. The model was seen to accurately predict the PSD. The model was then evaluated for various systems for a system of irregularly shaped particles: sand, plasma alumina and zinc dust. By irregularly shaped, it was implied that the shape of the particles within the system is not constant. The model was seen to reasonably predict the PSD, wherein the mean of the PSD was accurately predicted and the variance was over predicted. The model validity was then tested for a couple of systems of particles which are expected to be potential application areas of the presented model: paracetamol crystals and p-aminophenol crystals. The model was seen to accurately predict the PSD for these cases. The performance of the present model for these system was seen to be at par with the popular models presented by Li et. al. $(2005)^{2}$ and Worlischek et. al. (2005) ${ }^{5}$. These models are quite complex to implement for reasons discussed earlier as also require the specification of an additional parameter(s) for particle shape. In comparison the present model is rather simple (can be implemented using only simple spread sheeting tools) and does not require the specification of additional parameters.

\section{Acknowledgments}

The authors of the present work wish to thank Mettler Toledo for providing us with the measured Particle Size Distribution data obtained through image analysis using the Mettler Toledo PVM probe for a system of spherical, fully opaque, ceramic spheres. Authors are grateful to financial support for this work by CSIR through Mastflo [OLP3026] and Indus MAGIC [CSC123] projects. 


\section{Symbols \& Notation}

$P(x, y, D) \quad$ Probability of measuring a chord between $x$ and $y$ for a circle of diameter ' $D$ '

D Diameter of sphere/circle

$L_{i}, L_{i-1} \quad$ Upper and lower boundary locations respectively of the $\mathrm{i}^{\text {th }}$ bin

$N_{i} \quad$ Number of particles in the $i^{\text {th }}$ bin in the PSD

$M_{i} \quad$ Number of particles in the $i^{\text {th }}$ bin in the CLD

Fraction of the particles contained in the $m^{\text {th }}$ bin in the PSD measured as having chords of size

$A_{i, m} \quad$ belonging to the $i^{\text {th }}$ bin of the CLD

Fraction of the total particles measured as having chords of size belonging to the $i^{\text {th }}$ bin of the

$A_{i}$ CLD

$N_{T} \quad$ Total number of particles

$\mu \quad$ Model parameter referencing mean particle size

$\sigma^{2} \quad$ Model parameter referencing variance/spread of particle sizes

$B_{i} \quad i^{\text {th }}$ moment of the distribution considered

$X_{j} \quad$ The representative pivot location of the $j^{\text {th }}$ bin

$n_{1, l n} \quad$ Discretized length weighted logarithmic distribution

$n_{3, \ln } \quad$ Discretized volume weighted logarithmic distribution

$w_{s} \quad$ Logarithm of the ratio of the bin expansion; $w_{S}=\ln \left(\frac{L_{i}}{L_{i-1}}\right)$ 


\section{References}

[1] Li H, Grover M, Kawajiri Y, Rousseau R. Development of an empirical method relating crystal size distributions and FBRM measurements. Chem. Eng. Sci. 2013; 89:142-151

[2] Li M, Wilkinson D. Determination of non-spherical particle size distribution from chord length measurements. Part 1: Theoretical analysis. Chem. Eng. Sci. 2005; 60:3151-3265

[3] Li M, Wilkinson D. Determination of non-spherical particle size distribution from chord length measurements. Part 2: Experimental validation. Chem. Eng. Sci. 2005; 60:4992-5003

[4] Ruf A, Worlitschek J, Mazotti M. Modeling and Experimental analysis of PSD measurements through FBRM. Part. Part. Syst. Charact. 2000; 17:167-179

[5] Worlitschek J, Hocker T, Mazzotti M. Restoration of PSD from Chord Length Distribution Data using the Method of Projection onto Convex Sets. Part. Part. Syst. Charact. 2005; 22:81-98

[6] Agimelen O, Hamilton P, Haley I, Nordon S, Vasile M, Sefcik J, Mulholland A. Estimation of particle size distribution and aspect ratio of non-spherical particles from chord length distribution. Chem. Eng. Sci. 2015; 123:629-640

[7] Kail N, Marquardt W, Briesen H. Estimation of particle size distributions from focused beam reflectance measurements based on an optical model. Chem. Eng. Sci. 2009; 64(5):984-1000 


\section{List of Figures}

Figure 1: (a) Circle defined by a chord of length ' $x$ ' (b) Area considered to measure chord length between ' $x$ ' and ' $y$ '

Figure 2: Particle Size Distribution and Chord Length Distribution for a collection of monodisperse particles of $95 \mu m$ size

Figure 3: Algorithm to determine PSD from CLD

Figure 4: $\quad$ Procedure used to determine the PSD through image analysis

Figure 5: (a) Images obtained from calibration sample for the FBRM probe consisting of ceramic beads using a PVM probe (b) Comparison between the PSD measured using the particle widths and particle lengths obtained using PVM

Figure 6: (a) Comparison between normalized CLD measured using FBRM for a system consisting of ceramic beads and the normalized fitted CLD used for the model (b) Comparison between the normalized experimental PSD obtained for a system of ceramic beads obtained using PVM and the normalized model predicted PSD

Figure 7: (a) Comparison between the normalized CLD obtained using present model and the normalized experimentally measured CLD (using FBRM, Li et. al. (2005) ${ }^{3}$ ) for a system of ceramic bead particles (b) Comparison between the normalized PSD obtained using Image analysis (reported by Li et. al. $(2005)^{3}$ ), and the model predicted PSD’s using the 
present model and the model presented by Li et. al. (2005) ${ }^{3}$ for a system of ceramic bead particles

Figure 8: (a) Images taken under a micro scope for a sample of sand particles (b) Binary image obtained after image processing of image shown in (a)

Figure 9: (a) Comparison between the normalized square weighted CLD obtained using the present model, the normalized square weighted CLD measured using FBRM (averaged over 1 min) and the normalized CLD measured using FBRM (averaged over 1 min) for a sample of irregularly shaped sand particles (b) Comparison between the PSD obtained using the present model and the PSD obtained using Image Analysis for a sample of irregularly shaped sand particles

Figure 10: (a) Comparison between the normalized CLD obtained using present model and the normalized experimentally measured CLD (using FBRM, Li et. al. (2005) $)^{3}$ ) for a system of plasma alumina particles (b) Comparison between the normalized PSD obtained using Image analysis (reported by Li et. al. $(2005)^{3}$ ), and the model predicted PSD’s using the present model and the model presented by Li et. al. (2005) ${ }^{3}$ for a system of plasma alumina particles

Figure 11: (a) Comparison between the normalized CLD obtained using present model and the normalized experimentally measured CLD (using FBRM, Li et. al. $(2005)^{3}$ ) for a system of zinc dust particles (b) Comparison between the normalized PSD obtained using Image analysis (reported by Li et. al. $(2005)^{3}$ ), and the model predicted PSD’s using the present model and the model presented by Li et. al. $(2005)^{3}$ for a system of zinc dust particles

Figure 12: (a) Comparison between the restored CLD (Dotted line)(Worlitschek, Hocker and Mazotti (2005) $)^{5}$ ) and the derived CLD using the present model(b) A comparison between the discretized length-weighted logarithmic distributions obtained using the POCS method (POCS-Length) (Worlitschek, Hocker and Mazotti (2005) ${ }^{5}$ ), the derived PSD in the present model (PSD-Length) and the TSI Aerosizer (Worlitschek, Hocker and 
Mazotti (2005) $)^{5}$ ) (c) A comparison between the discretized volume-weighted logarithmic distributions obtained using the POCS method (POCS-Volume) (Worlitschek, Hocker and Mazotti (2005)5), the derived PSD in the present model (PSD-Volume) and laser diffraction (Worlitschek, Hocker and Mazotti $(2005)^{5}$ )

Figure 13: (a) Raw micrograph of p-Aminophenol crystals (b) Comparison between the normalized squared CLD obtained using present model and the normalized experimentally measured (using FBRM) squared and non-squared CLD for a system of p-Aminophenol crystals (c) Comparison between the normalized PSD obtained using Image analysis and the model predicted PSD for a system of p-Aminophenol crystals 
(a)

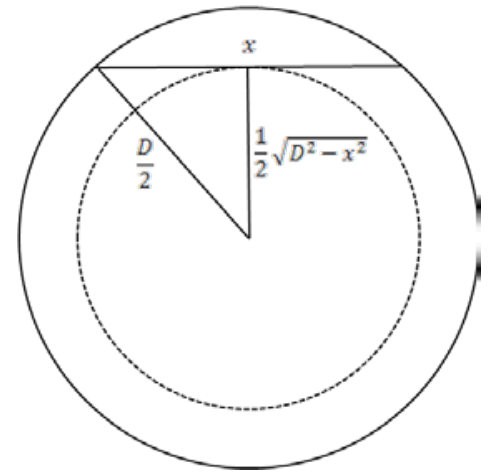

(b)

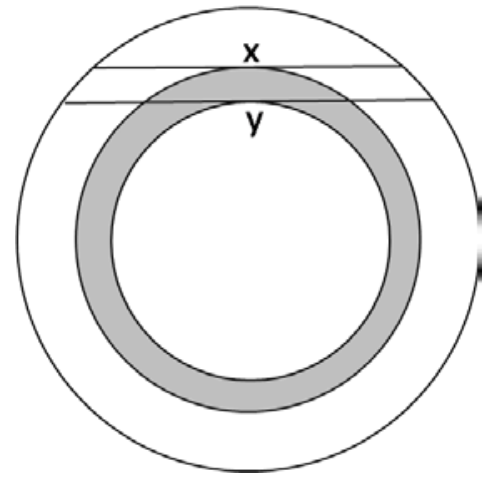

Figure 1: (a) Circle defined by a chord of length $x$ (b) Area considered to measure chord length between $x$ and $y$ 


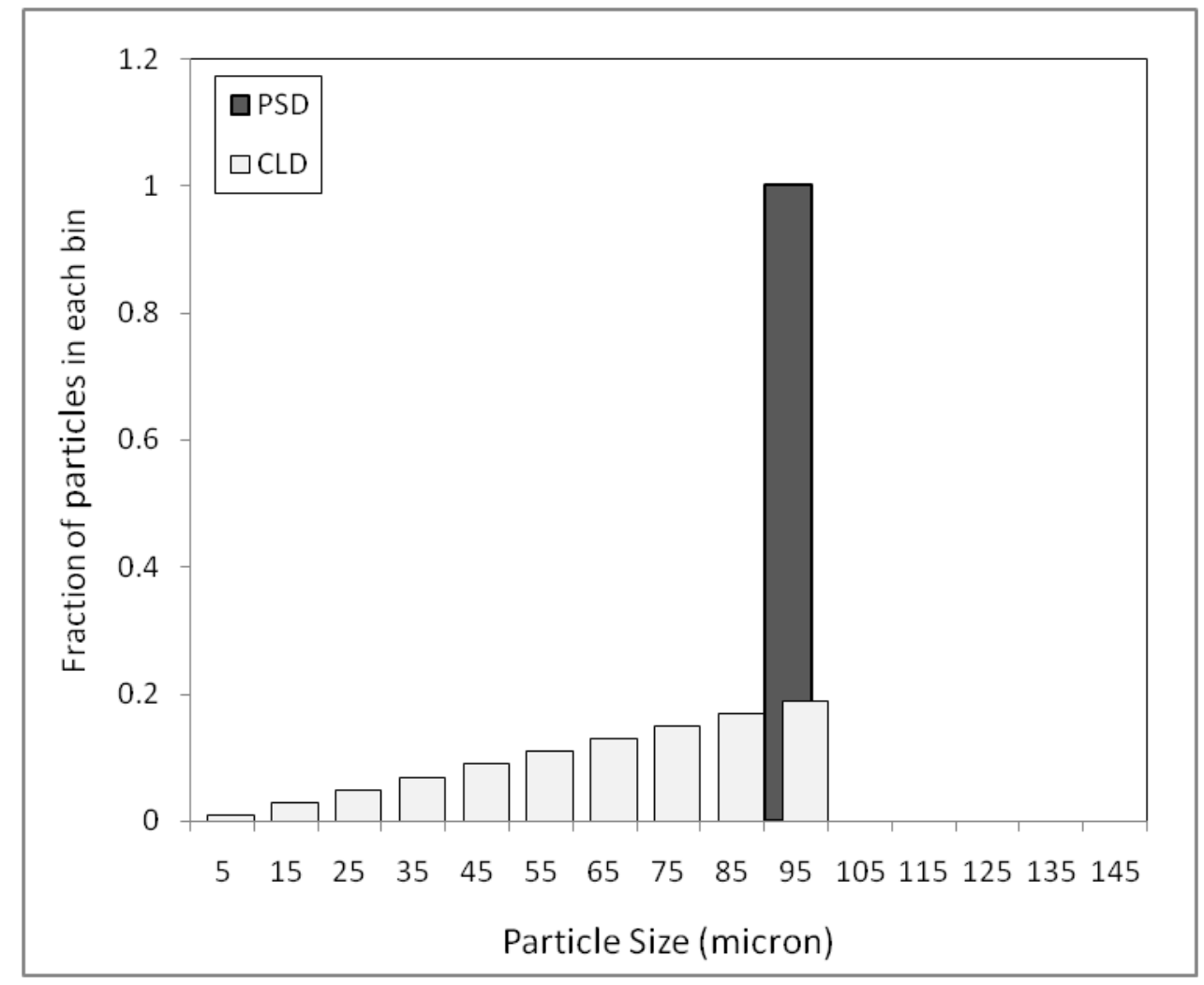

Figure 2: Particle Size Distribution and Chord Length Distribution for a collection of monodisperse particles of $95 \mu m$ size 


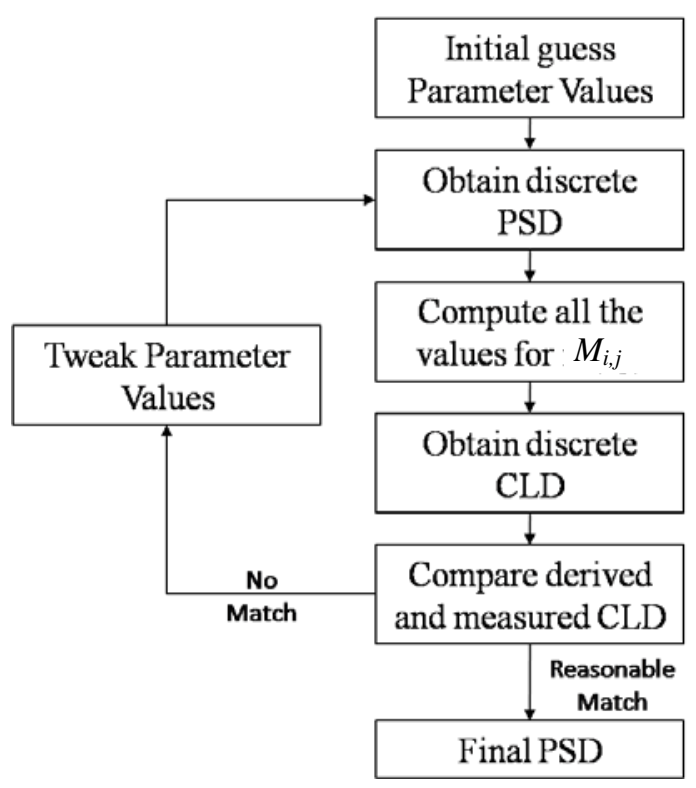

Figure 3: Algorithm to determine PSD from CLD 


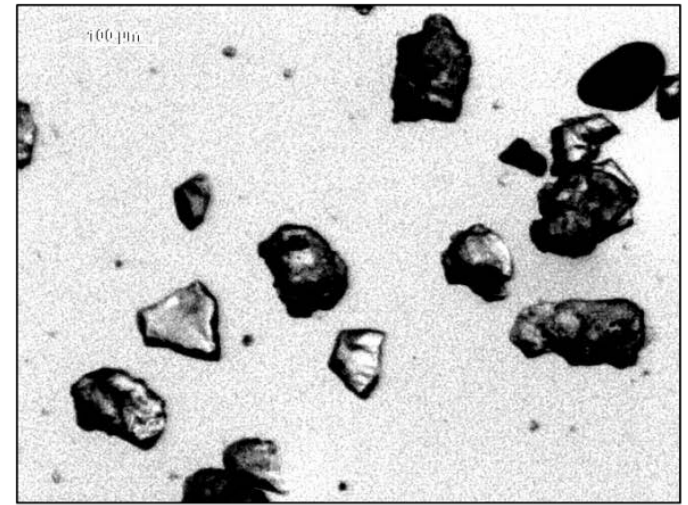

Raw Microscope Images

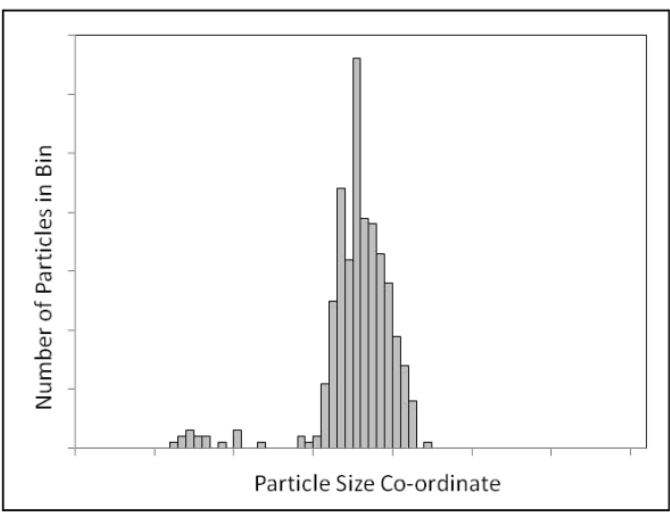

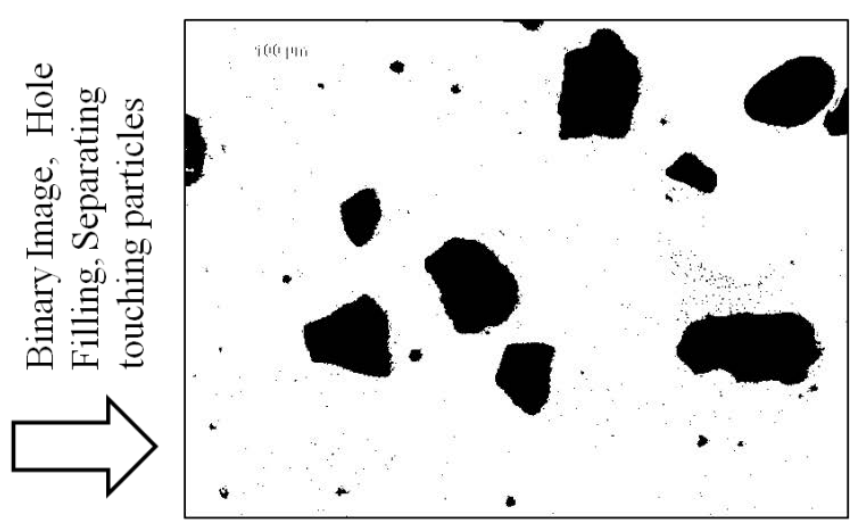

Noise/Speck removal, Removing edge particles, Particle edge detection

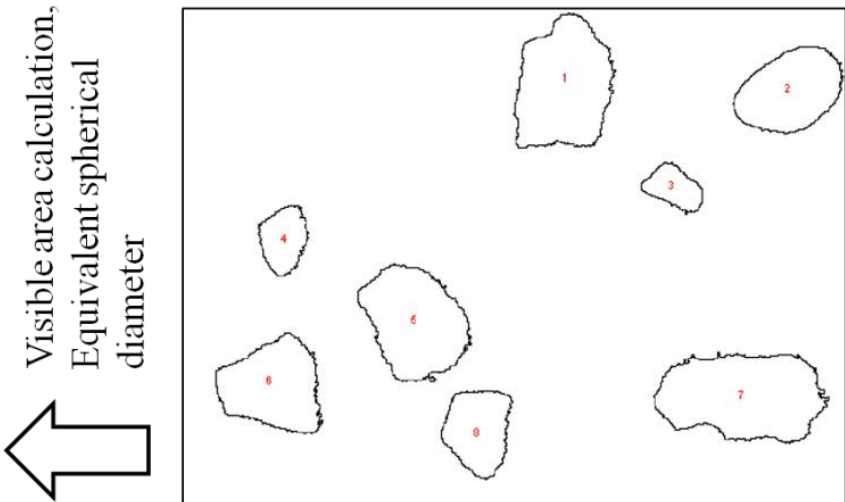

Particle Size Distribution

Figure 4: Procedure used to determine the PSD through image analysis 
(a)

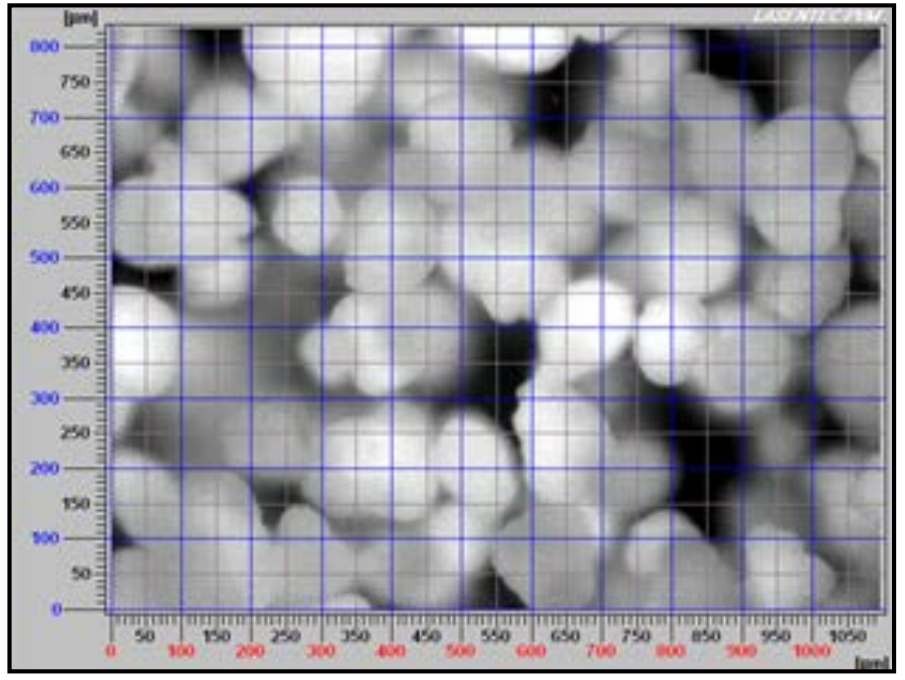

(b)

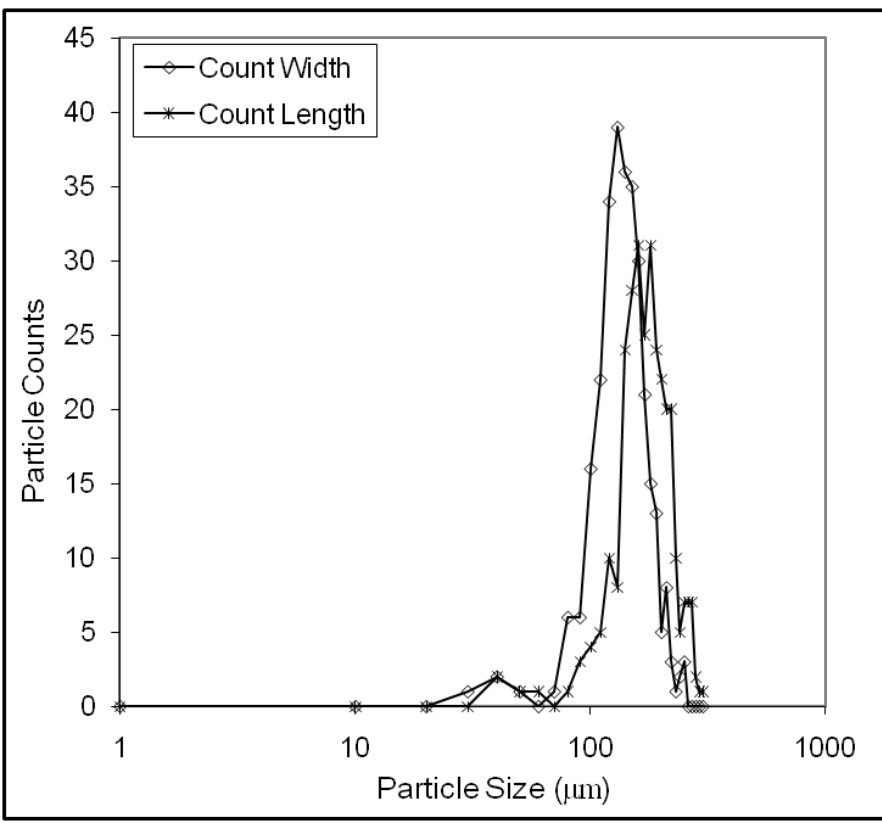

Figure 5: (a) Images obtained from calibration sample for theFBRM probe consisting of ceramic beads using a PVM probe (b) Comparison between the PSD measured using the particle widths and particle lengths obtained using PVM 


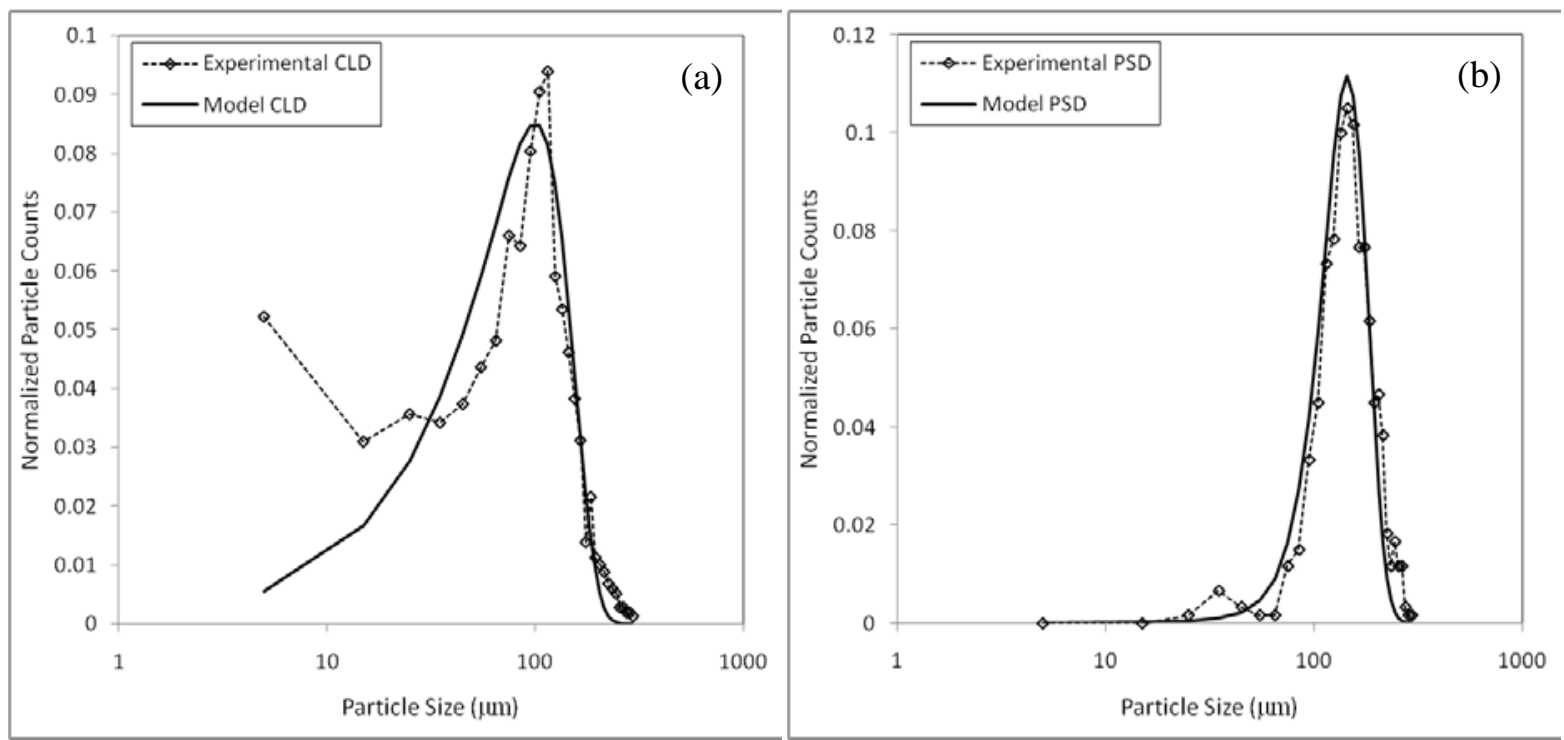

Figure 6: (a) Comparison between normalized CLD measured using FBRM for a system consisting of ceramic beads and the normalized fitted CLD used for the model (b) Comparison between the normalized experimental PSD obtained for a system of ceramic beads obtained using PVM and the normalized model predicted PSD 


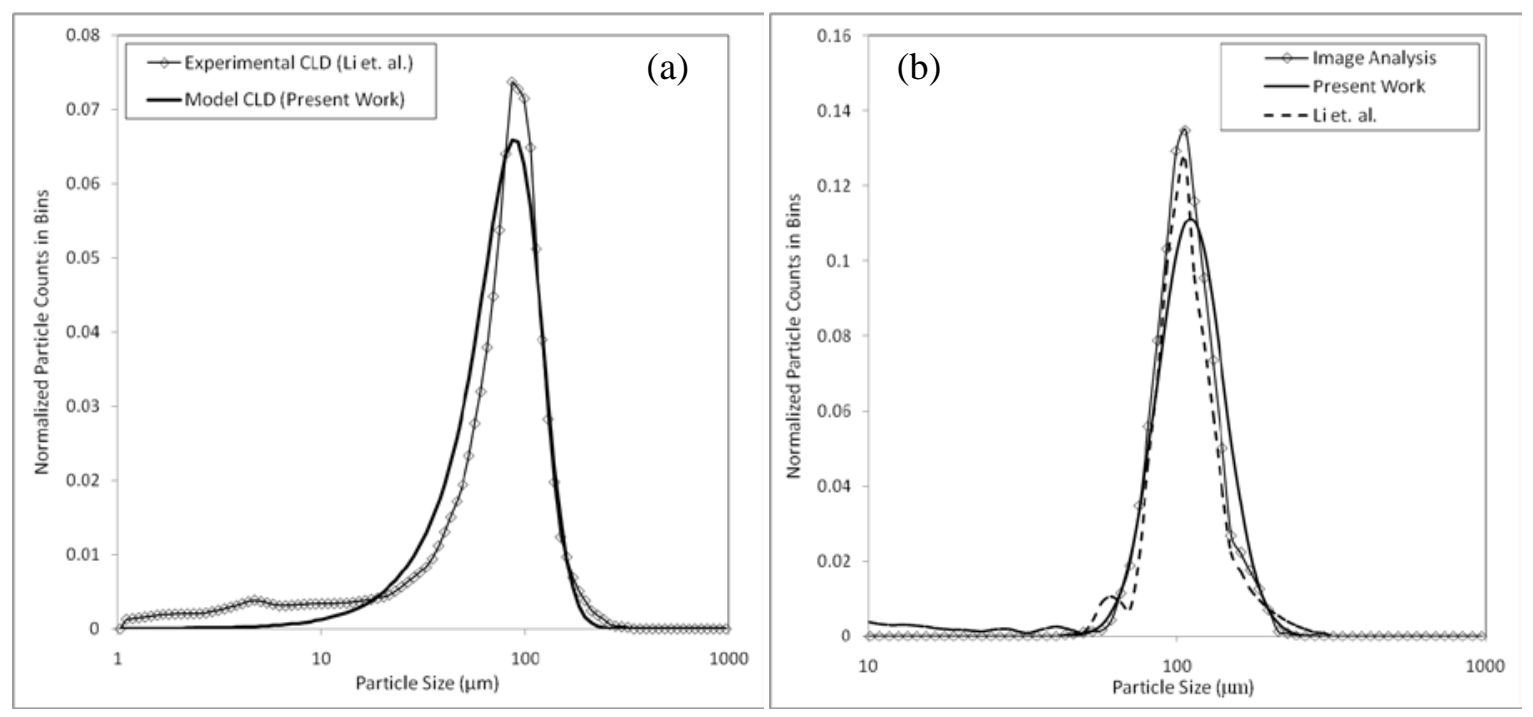

Figure 7: (a) Comparison between the normalized CLD obtained using present model and the normalized experimentally measured CLD (using FBRM, Li et. al. (2005) ${ }^{3}$ ) for a system of ceramic bead particles (b) Comparison between the normalized PSD obtained using Image analysis (reported by Li et. al. $(2005)^{3}$ ), and the model predicted PSD’s using the present model and the model presented by Li et. al. (2005) ${ }^{3}$ for a system of ceramic bead particles 


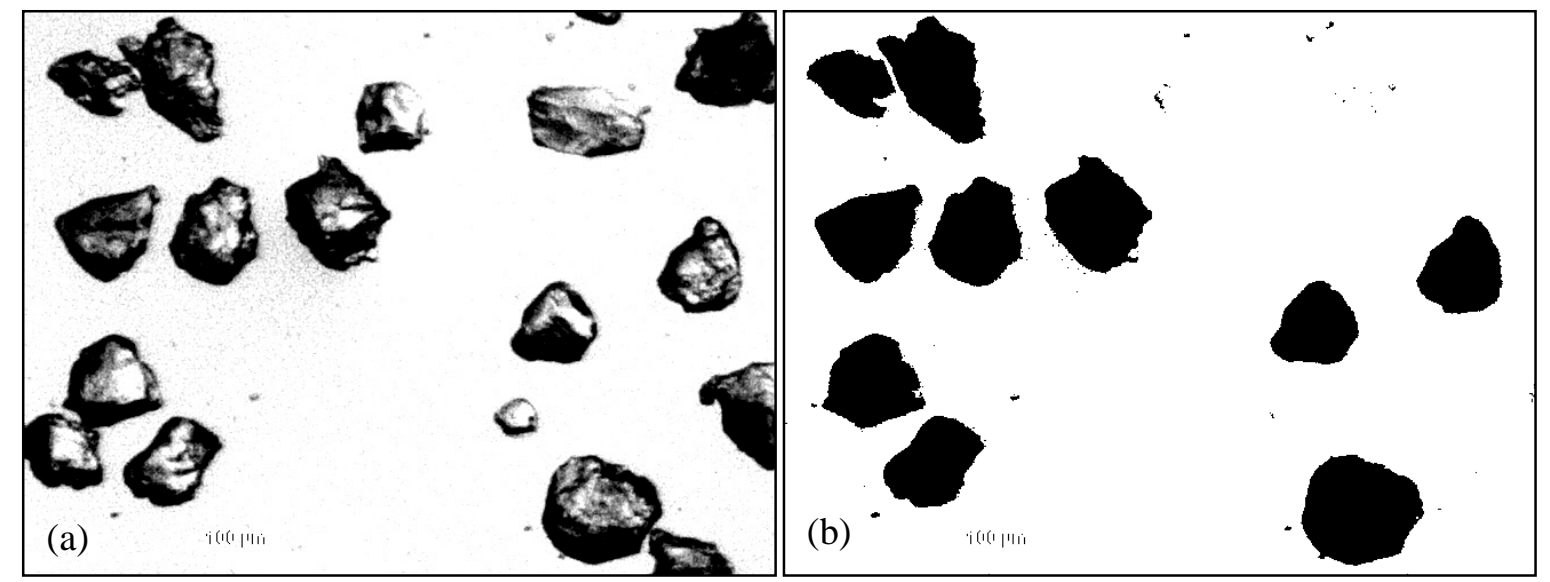

Figure 8: (a) Images taken under a micro scope for a sample of sand particles (b) Binary image obtained after image processing of image shown in (a) 


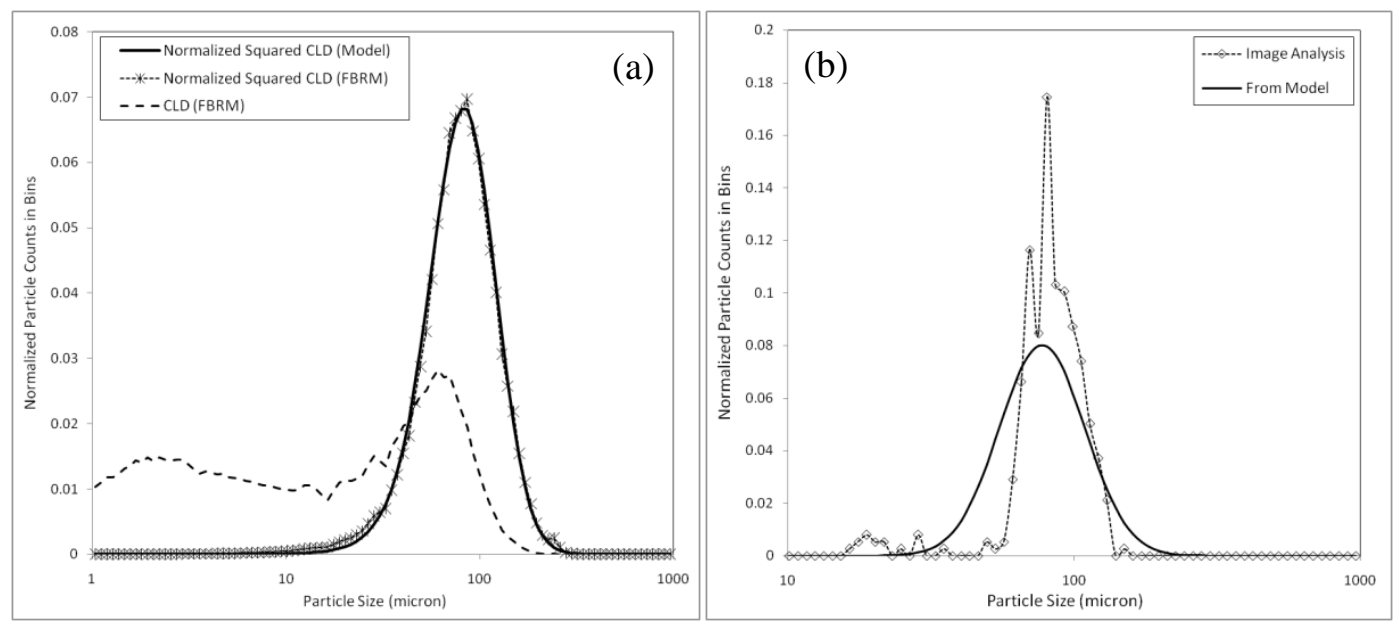

Figure 9: (a) Comparison between the normalized square weighted CLD obtained using the present model, the normalized square weighted CLD measured using FBRM (averaged over 1 min) and the normalized CLD measured using FBRM (averaged over $1 \mathrm{~min}$ ) for a sample of irregularly shaped sand particles (b) Comparison between the PSD obtained using the present model and the PSD obtained using Image Analysis for a sample of irregularly shaped sand particles 

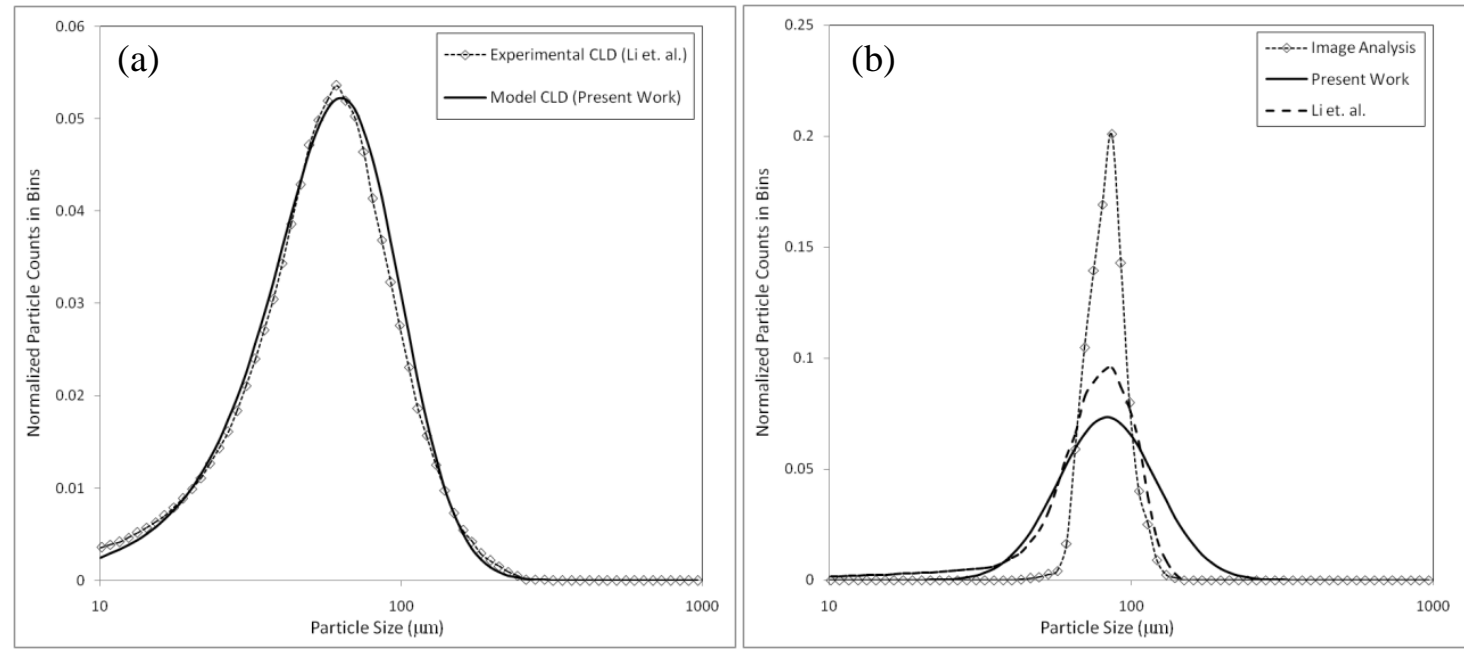

Figure 10: (a) Comparison between the normalized CLD obtained using present model and the normalized experimentally measured CLD (using FBRM, Li et. al. (2005) ${ }^{3}$ ) for a system of plasma alumina particles (b) Comparison between the normalized PSD obtained using Image analysis (reported by Li et. al. $(2005)^{3}$ ), and the model predicted PSD's using the present model and the model presented by Li et. al. (2005) ${ }^{3}$ for a system of plasma alumina particles 


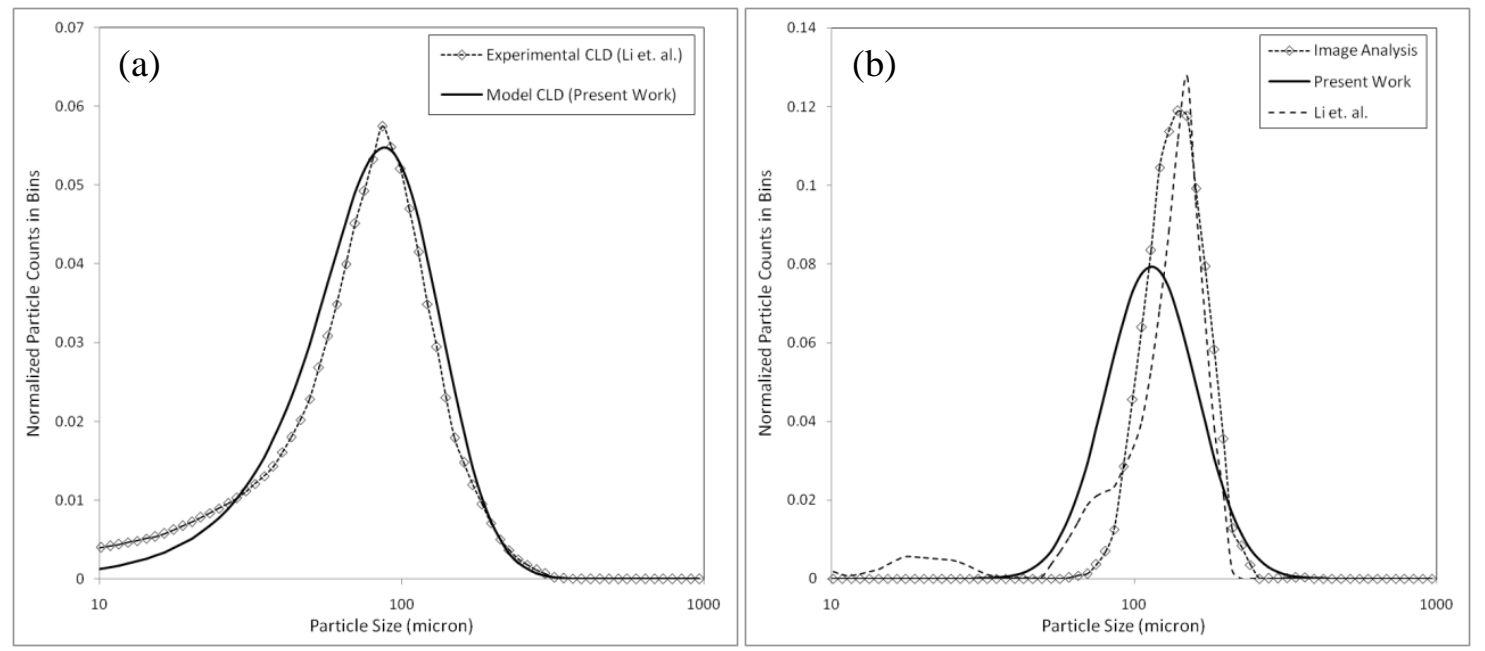

Figure 11: (a) Comparison between the normalized CLD obtained using present model and the normalized experimentally measured CLD (using FBRM, Li et. al. (2005) ${ }^{3}$ ) for a system of zinc dust particles (b) Comparison between the normalized PSD obtained using Image analysis (reported by Li et. al. $\left.(2005)^{3}\right)$, and the model predicted PSD’s using the present model and the model presented by Li et. al. (2005) ${ }^{3}$ for a system of zinc dust particles 

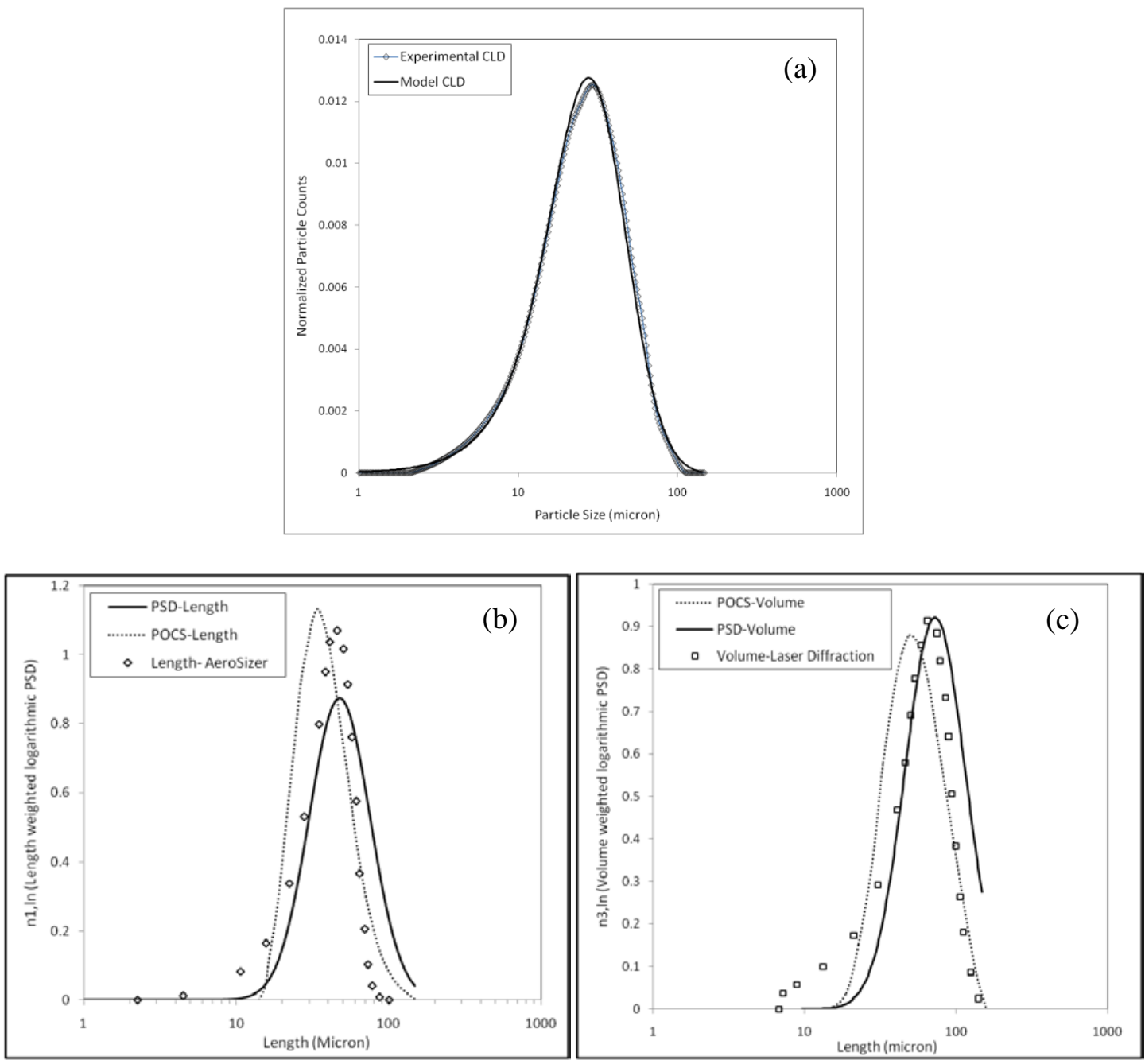

Figure 12: (a) Comparison between the restored CLD (Dotted line)(Worlitschek, Hocker and Mazotti $\left.(2005)^{5}\right)$ and the derived CLD using the present model(b) A comparison between the discretized length-weighted logarithmic distributions obtained using the POCS method (POCS-Length) (Worlitschek, Hocker and Mazotti (2005) $)$, the derived PSD in the present model (PSD-Length) and the TSI Aerosizer (Worlitschek, Hocker and Mazotti (2005) $)^{5}$ ) (c) A comparison between the discretized volume-weighted logarithmic distributions obtained using the POCS method (POCSVolume) (Worlitschek, Hocker and Mazotti (2005) $)^{5}$, the derived PSD in the present model (PSDVolume) and laser diffraction (Worlitschek, Hocker and Mazotti (2005) ${ }^{5}$ ) 
(a)
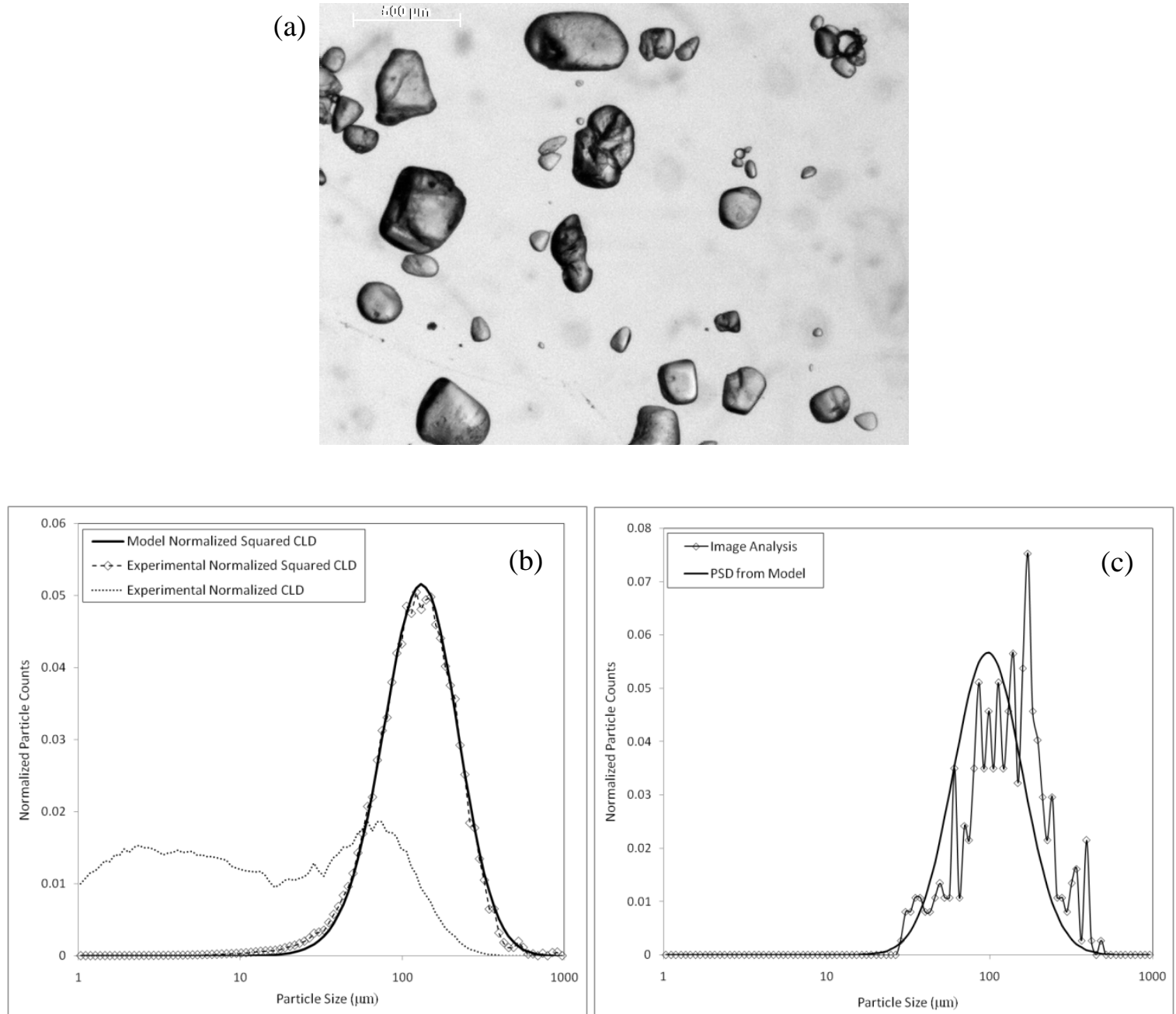

Figure 13: (a) Raw micrograph of p-Aminophenol crystals (b) Comparison between the normalized squared CLD obtained using present model and the normalized experimentally measured (using FBRM) squared and non-squared CLD for a system of p-Aminophenol crystals (c) Comparison between the normalized PSD obtained using Image analysis and the model predicted PSD for a system of p-Aminophenol crystals 


\section{List of Tables}

Table 1: The expected value for the mean and the standard deviation for the different distributions considered in the present study 


\begin{tabular}{|c|c|c|c|c|c|}
\hline \multicolumn{3}{|c|}{ PVC Sample (Spherical) } & \multicolumn{3}{|c|}{ Zinc Dust (Li et. al.) (Irregular) } \\
\hline & Expected Value & Std. Dev & & Expected Value & Std. Dev \\
\hline Image Analysis & 156.33 & 43.57 & Li et. al. & 119.45 & 48.05 \\
\hline Present Model & 150.00 & 33.33 & Image Analysis & 140.29 & 31.25 \\
\hline & & & Present Model & 121.26 & 43.42 \\
\hline \multicolumn{6}{|c|}{ Ceramic Beads (Li et. al.) (Spherical) } \\
\hline Li et. al. Model & 98.01 & 43.58 & \multicolumn{3}{|c|}{ Para-Amino Phenol (Regular) } \\
\hline Image Analysis & 109.20 & 25.97 & Image Analysis & 144.01 & 81.39 \\
\hline Present Model & 113.63 & 28.55 & Present Model & 109.97 & 56.76 \\
\hline \multicolumn{3}{|c|}{ Sand (Irregular) } & \multicolumn{3}{|c|}{ Paracetamol (Length weighted distribution) } \\
\hline Image Analysis & 84.21 & 21.21 & POCS - Length & 40.76 & 18.36 \\
\hline \multirow[t]{2}{*}{ Present Model } & 81.88 & 29.00 & TSI Aero & 38.16 & 15.45 \\
\hline & & & Present Model & 51.96 & 23.64 \\
\hline \multicolumn{3}{|c|}{ Plasma Alumina (Li et. al.) (Irregular) } & \multicolumn{3}{|c|}{ Paracetamol (Volume weighted distribution) } \\
\hline Li et. al. Model & 75.07 & 25.27 & POCS - Volume & 57.35 & 25.04 \\
\hline Image Analysis & 83.81 & 12.78 & Laser Diffraction & 56.48 & 26.70 \\
\hline Present Model & 89.24 & 34.69 & Present Model & 74.10 & 28.76 \\
\hline
\end{tabular}

Table 1: The expected value for the mean and the standard deviation for the different distributions considered in the present study (all values are considered in $\mu \mathrm{m}$ ) 